\title{
Dirichlet BVP for the Second Order Nonlinear Ordinary Differential Equations at Resonance
}

\section{Sulkhan Mukhigulashvili ${ }^{a}$ and Mariam Manjikashvili ${ }^{b}$}

${ }^{a}$ Faculty of Business and Management, Brno University of Technology Kolejni 2906/4, 61200 Brno, Czech Republic

${ }^{b}$ Faculty of Business, Technology and Education, Ilia State University

I. Chavchavadze Str. No.32, 0179 Tbilisi, Georgia

E-mail(corresp.): smukhig@gmail.com

E-mail: manjikashvilimary@gmail.com

Received April 23, 2019; revised September 16, 2019; accepted September 17, 2019

Abstract. Landesman-Lazer's type efficient sufficient conditions are established for the solvability of the Dirichlet problem

$$
u^{\prime \prime}(t)=p(t) u(t)+f(t, u(t))+h(t), \text { for } a \leq t \leq b, u(a)=0, u(b)=0,
$$

where $h, p \in L([a, b] ; R)$ and $f$ is the $L([a, b] ; R)$ Caratheodory function, in the case where the linear problem $u^{\prime \prime}(t)=p(t) u(t), u(a)=0, u(b)=0$ has nontrivial solutions. The results obtained in the paper are optimal in the sense that if $f \equiv 0$, i.e., when nonlinear equation turns to the linear equation, from our results follows the first part of Fredholm's theorem.

Keywords: nonlinear ordinary differential equation, Dirichlet problem at resonance.

AMS Subject Classification: 34B15; 34C15; 34C25.

\section{Introduction}

Consider on the interval $I=[a, b]$ the second order nonlinear ordinary differential equation

$$
u^{\prime \prime}(t)=p(t) u(t)+f(t, u(t))+h(t)
$$

with the boundary conditions

$$
u(a)=0, \quad u(b)=0,
$$


where $h, p \in L(I ; R)$ and $f \in K(I \times R ; R)$. By a solution of the problem (1.1)(1.2) we understand a function $u \in \widetilde{C}^{\prime}(I, R)$, which satisfies equation (1.1) almost everywhere on $I$ and satisfies conditions (1.2).

Along with (1.1)-(1.2) we consider the homogeneous problem

$$
\begin{aligned}
w^{\prime \prime}(t) & =p(t) w(t) \quad \text { for } \quad t \in I, \\
w(a) & =0, \quad w(b)=0 .
\end{aligned}
$$

At present, the foundations of the general theory of two-point boundary value problems are already laid and problems of this type are studied by many authors and investigated in detail. But primarily is studied the case when the homogeneous problem (1.3)-(1.4) has only a trivial solution i.e., the non resonance case (see for instance $[2,3,5,11]$ and references therein).

The case when problem (1.3)-(1.4) has the nontrivial solution is still little investigated and the majority of the authors study the case when $p$ is constant function and this constant is the first eigenvalue of linear problem (see, for instance, $[1,3,4,6,7,8,9,10,12]$ and references therein), i.e., when problem (1.1)-(1.2) and equation (1.3) are of the following type

$$
\begin{aligned}
u^{\prime \prime}(t) & =-\lambda^{2} u(t)+f(t, u(t))+h(t) \quad \text { for } \quad t \in[0, \pi], \\
u(0) & =0, \quad u(\pi)=0,
\end{aligned}
$$

and $w^{\prime \prime}(t)=-\lambda^{2} w(t)$ for $t \in[0, \pi]$, respectively, and $\lambda=1$. Some interesting results of this type are presented for example in [3] (see the Theorems 2.5-2.10). Also the rare exception is the paper [7], where the authors study problem (1.5)(1.6) for $\lambda \geq 1$.

In the present article we establish the Landesman-Lazer's type conditions of the solvability of problem (1.1)-(1.2), when the function $p \in L(I ; R)$ is not necessarily constant, under the assumption that the nontrivial solution of homogeneous problem (1.3)-(1.4) has the zeros in the open interval $] a, b[$ (for problem (1.5)-(1.6) this is the case $\lambda \geq 2$.) The obtained theorems generalize deeply the results from the above mentioned articles, and give the principal improvement of the our previous results from the paper [13] (see Theorems 2.3, $2.4,2.5$ ). Also the obtained results are optimal in the sense that if $f \equiv 0$, i.e., when the equation (1.1) turns to the linear equation, from our results follows the first part of Fredholm's theorem (see Remark 6).

The following notation is used throughout the paper: $N$ is the set of all natural numbers; $R$ is the set of all real numbers, $R_{+}=[0,+\infty[; C(I ; R)$ is the Banach space of continuous functions $u: I \rightarrow R$ with the norm $\|u\|_{C}=$ $\max \{|u(t)|: t \in I\} ; \widetilde{C}^{\prime}(I ; R)$ is the set of functions $u: I \rightarrow R$ which are absolutely continuous together with their first derivatives; $L(I ; R)$ is the Banach space of Lebesgue integrable functions $p: I \rightarrow R$ with the norm $\|p\|_{L}=$ $\int_{a}^{b}|p(s)| d s ; \quad K(I \times R ; R)$ is the set of functions $f: I \times R \rightarrow R$ satisfying the Carathéodory conditions, i.e., $f(\cdot, x): I \rightarrow R$ is a measurable function for all $x \in R, f(t, \cdot): R \rightarrow R$ is a continuous function for almost all $t \in I$, and for arbitrary $r \in R_{+}$

$$
f^{*}(t, r)=\sup \{|f(t, x)|:|x| \leq r\} \in L\left(I, R_{+}\right) .
$$


Also having the function $x: I \rightarrow R$, we put:

$$
[x(t)]_{+}=(|x(t)|+x(t)) / 2, \quad[x(t)]_{-}=(|x(t)|-x(t)) / 2 .
$$

Remark 1. a. The dimension of the space of the nontrivial solutions of problem (1.3)-(1.4) is one; b. If $w_{1}$ and $w$ are the nonzero solutions of problem (1.3)(1.4), then

$$
w_{1}(t)=\beta w(t) \text { for } \quad t \in I,
$$

where $\beta=-\left\|w_{1}\right\|_{C} /\|w\|_{C}$ or $\beta=\left\|w_{1}\right\|_{C} /\|w\|_{C}$.

From Remark 1 it is clear that if $w$ is an arbitrary nonzero solution of problem (1.3)-(1.4) then the notation $N_{p} \equiv\{t \in] a, b[: w(t)=0\}$ is correct.

Definition 1. Let $f \in K(I \times R ; R)$ and $A=\left\{t_{1}, \cdots, t_{k}\right\}$ be a finite subset of $I$. Then we say $f \in E(A)$ if for an arbitrary neighbourhood $U(A)$ of the set $A$, and $r \in] 0,+\infty\left[\right.$, there exists $\lambda_{1}>0$ such that

$$
\int_{U^{\prime}(A) \backslash U_{\lambda}}|f(s, x)| d s-\int_{U_{\lambda}}|f(s, x)| d s \geq 0 \quad \text { for } \quad|x| \geq r, \lambda \leq \lambda_{1},
$$

where $U^{\prime}(A)=I \cap U(A)$, and $U_{\lambda}=I \cap\left(\cup_{j=1}^{k}\left[t_{j}-\lambda, t_{j}+\lambda\right]\right)$.

Remark 2. In example 1.1 of [13], for given set $A \subset I$ is constructed $f \in$ $K(I \times R ; R)$ such, that $f \notin E(A)$.

Remark 3. It is clear that if $f(t, x) \stackrel{\text { def }}{=} f_{0}(t) g_{0}(x)$, where $f_{0} \in L(I ; R)$ and $g_{0} \in C(R ; R)$, then $f \in E(A)$ for an arbitrary finite $A \subset I$.

\section{Main results}

In this paper we study problem (1.3)-(1.4) only under the assumption that $N_{p} \neq \emptyset$. The second order ordinary differential equations under different two point boundary conditions for the case $N_{p}=\emptyset$, are studied in our articles [13] and [14].

In the following propositions we always suppose that $i \in\{0,1\}$ takes one of the values 0 or 1 .

Theorem 1. Let $i \in\{0,1\}, r>0, f \in E\left(N_{p}\right)$, functions $f^{+}, f^{-} \in L\left(I ; R_{+}\right)$be such that

$$
\begin{gathered}
(-1)^{i} f(t, x) \leq-f^{-}(t) \quad \text { for } \quad x \leq-r, \quad t \in I, \\
f^{+}(t) \leq(-1)^{i} f(t, x) \quad \text { for } \quad x \geq r, \quad t \in I,
\end{gathered}
$$

and condition

$$
\lim _{\rho \rightarrow+\infty} \frac{1}{\rho} \int_{a}^{b} f^{*}(s, \rho) d s=0
$$


holds. Let moreover $w$ be an arbitrary nonzero solution of problem (1.3)-(1.4) and there exists $\varepsilon>0$ such that

$$
\begin{aligned}
& -\int_{a}^{b}\left(f^{+}(s)[w(s)]_{-}+f^{-}(s)[w(s)]_{+}\right) d s+\varepsilon \gamma_{r}\|w\|_{C} \leq(-1)^{i+1} \int_{a}^{b} h(s) w(s) d s \\
& \quad \leq \int_{a}^{b}\left(f^{-}(s)[w(s)]_{-}+f^{+}(s)[w(s)]_{+}\right) d s-\varepsilon \gamma_{r}\|w\|_{C}
\end{aligned}
$$

where $\gamma_{r}=\int_{a}^{b} f^{*}(s, r) d s$. Then problem (1.1)-(1.2) has at least one solution.

Remark 4. In view of item a. of Remark 1 it is clear that if $f \not \equiv 0$, then condition $\left(2.2_{i}\right)$ in Theorem 1 can be replaced by the condition

$$
\begin{gathered}
-\int_{a}^{b}\left(f^{+}(s)[w(s)]_{-}+f^{-}(s)[w(s)]_{+}\right) d s<(-1)^{i+1} \int_{a}^{b} h(s) w(s) d s \\
<\int_{a}^{b}\left(f^{-}(s)[w(s)]_{-}+f^{+}(s)[w(s)]_{+}\right) d s .
\end{gathered}
$$

Remark 5. If $\tilde{f}(t)=\min \left\{f^{+}(t), f^{-}(t)\right\}$ then

$$
\int_{a}^{b} \tilde{f}(s)|w(s)| d s \leq \int_{a}^{b}\left(f^{ \pm}(s)[w(s)]_{-}+f^{\mp}(s)[w(s)]_{+}\right) d s,
$$

and therefore condition $\left(2.2_{i}\right)$ will be fulfilled if

$$
\left|\int_{a}^{b} h(s) w(s) d s\right| \leq \int_{a}^{b} \widetilde{f}(s)|w(s)| d s-\varepsilon \gamma_{r}\|w\|_{C} .
$$

Consequently condition $\left(2.2_{i}\right)$ can be replaced by the last inequality.

Remark 6. From inequality (2.3) is evident that if $f \equiv 0$, then $f \equiv f^{+} \equiv f^{-} \equiv$ $0, \gamma_{r}=0$, and Theorem 1 turns to the first part of Fredholm's Theorem.

Theorem 2. Let $i \in\{0,1\}, r>0$, and the conditions

$$
(-1)^{i} f(t, x) \operatorname{sgn} x \geq 0 \quad \text { for } \quad|x| \geq r, \quad t \in I,
$$

and (2.2) be satisfied. Let moreover there exist sets $I^{+}, I^{-} \subset I$ such, that

$$
\lim _{x \rightarrow \pm \infty}|f(t, x)|=+\infty \quad \text { uniformly on } I^{ \pm},
$$

and for an arbitrary solution $w$ of problem (1.3)-(1.4) the inequalities

$$
\int_{I^{+}}[w(s)]_{+} d s+\int_{I^{-}}[w(s)]_{-} d s \neq 0, \quad \int_{I^{+}}[w(s)]_{-} d s+\int_{I^{-}}[w(s)]_{+} d s \neq 0,
$$

hold. Then for an arbitrary $h \in L(I ; R)$ problem $(1.1)-(1.2)$ has at least one solution. 
If $I_{1}=I^{+} \cap I^{-}$is the set of positive measure, then condition (2.5) automatically holds, and from Theorem 2 it follows

Corollary 1. Let $i \in\{0,1\}, r>0$, and the function $f \in E\left(N_{p}\right)$ be such that the conditions (2.2) and (2.4) are satisfied. Let moreover there exist such a set $I_{1} \subset I$ of positive measure that

$$
\lim _{x \rightarrow \pm \infty}|f(t, x)|=+\infty \quad \text { uniformly on } I_{1} .
$$

Then for an arbitrary $h \in L(I ; R)$ problem (1.1)-(1.2) has at least one solution. If we note that from condition (2.6) follows validity of condition (2.4), from Corollary 1 we get

Corollary 2. Let $i \in\{0,1\}$, and the function $f \in E\left(N_{p}\right)$ be such that the condition (2.2) is satisfied, and

$$
(-1)^{i} \lim _{x \rightarrow \pm \infty} f(t, x)= \pm \infty \quad \text { uniformly on } I .
$$

Then for an arbitrary $h \in L(I ; R)$ problem (1.1)-(1.2) has at least one solution.

Also in view of Remark 3 from the Corollary 1 immediately follows

Corollary 3. Let $i \in\{0,1\}, f(t, x) \stackrel{\text { def }}{\equiv} f_{0}(t) g(x)$ where the functions $f_{0} \in$ $L\left(I ; R_{+}\right)$, and $g \in C(R ; R)$, be such that

$$
\begin{aligned}
& \int_{a}^{b} f_{0}(s) d s \neq 0, \quad \lim _{|x| \rightarrow+\infty} \frac{g(x)}{x}=0, \\
& (-1)^{i} \lim _{x \rightarrow+\infty} g(x)=+\infty, \quad(-1)^{i} \lim _{x \rightarrow-\infty} g(x)=-\infty .
\end{aligned}
$$

Then for an arbitrary $h \in L(I ; R)$ problem (1.1)-(1.2) has at least one solution.

Example 1. From Corollary 3 it follows that the equation

$$
u^{\prime \prime}(t)=-\lambda^{2} u(t)+\sigma|u(t)|^{\alpha} \operatorname{sgn} u(t)+h(t) \quad \text { for } \quad 0 \leq t \leq \pi
$$

under the boundary conditions (1.6), where $\sigma \in\{-1,1\}, \lambda \geq 2$, and $\alpha \in] 0,1[$, has at least one solution for an arbitrary $h \in L([0, \pi], R)$.

Remark 7. For problem (1.5)-(1.6) when $\lambda \geq 2$, the proved propositions are valid with $w(t)=\beta \sin \lambda t(\beta \in R)$, and

$$
N_{p}=N_{\lambda^{2}}=\{\pi n / \lambda: n=1, \ldots, \lambda-1\} .
$$

Example 2. From Remark 5 , with $f^{+} \equiv f^{-} \equiv 1-\delta$ for arbitrary $\left.\delta \in\right] 0,1 / 2[$, it follows that the equation

$$
u^{\prime \prime}(t)=-\lambda^{2} u(t)+\frac{\sigma|u(t)|^{\alpha}}{1+|u(t)|^{\alpha}} \operatorname{sgn} u(t)+h(t) \quad \text { for } \quad 0 \leq t \leq \pi
$$

under the boundary conditions (1.6), where $\sigma \in\{-1,1\}, \lambda \geq 2$, and $\alpha>0$, is solvable if $|h(t)|<1$ on $[0, \pi]$, or $\int_{0}^{\pi} h(s) \sin \lambda s d s=0$. 


\section{Auxiliary propositions}

Let $A, B \subset I$ be measurable sets, $N_{p}=\left\{t_{1}, \cdots, t_{k}\right\}\left(a<t_{j}<t_{j+1}<b\right), \alpha>0$, $w \in C(I, R)$ and

$$
\begin{aligned}
& \left.U_{\alpha} \stackrel{\text { def }}{\equiv} \cup_{j=1}^{k}\right] t_{j}-\alpha, t_{j}+\alpha[ \\
& \mathbb{I}(A, B, x, w) \stackrel{\text { def }}{=} \int_{A \backslash B}\left|f_{1}(s, x) w(s)\right| d s-\int_{B}\left|f_{1}(s, x) w(s)\right| d s \text { for } x \in R .
\end{aligned}
$$

Then the following lemma is true

Lemma 1. Let $f_{1} \in E\left(N_{p}\right)$, and $w$ be a nonzero solution of problem (1.3)(1.4). Then for an arbitrary $\delta \in] 0,1 / 2 \min \left\{t_{1}-a, b-t_{k}\right\}[$, there exists $\gamma \in] 0, \delta[$ such that

$$
\mathbb{I}\left(I_{\delta}, U_{\gamma}, x, w\right) \geq \int_{I_{\delta} \backslash U_{\delta}}\left|f_{1}(s, x) w(s)\right| d s \quad \text { for } \quad|x| \geq r,
$$

where $I_{\delta}=[a+\delta, b-\delta]$.

Proof. From definition 1 it follows the existence of such positive $\gamma_{1}<\delta$ that

$$
\mathbb{I}\left(U_{\delta}, U_{\gamma_{1}}, x, 1\right) \geq 0 \quad \text { for } \quad|x| \geq r .
$$

Let now $w_{0}$ be a solution of problem (1.3)-(1.4) and $\left\|w_{0}\right\|_{C}=1$. Then from the inclusion $N_{p} \subset U_{\gamma_{1}} \subset I_{\delta}$ it is evident that there exist positive numbers $\gamma, \beta_{0}$, such that

$$
U_{\gamma} \subset H_{\beta_{0}} \stackrel{\text { def }}{\equiv}\left\{t \in I_{\delta}:\left|w_{0}(t)\right| \leq \beta_{0}\right\} \subset U_{\gamma_{1}} .
$$

Then the inequality $\gamma_{1}<\delta$ and (3.3) imply inclusion $H_{\beta_{0}} \subset U_{\delta}$, and the relations

$$
\begin{aligned}
& \left|w_{0}(t)\right|>\beta_{0} \text { for } t \in U_{\delta} \backslash H_{\beta_{0}}, \\
& I_{\delta} \backslash H_{\beta_{0}}=\left(I_{\delta} \backslash U_{\delta}\right) \cup\left(U_{\delta} \backslash H_{\beta_{0}}\right),
\end{aligned}
$$

hold. From the last expression, Remark 1, and (3.3), (3.4), we have

$$
\begin{aligned}
& \frac{1}{\|w\|_{C}} \mathbb{I}\left(I_{\delta}, U_{\gamma}, x, w\right)=\mathbb{I}\left(I_{\delta}, U_{\gamma}, x, w_{0}\right) \geq \mathbb{I}\left(I_{\delta}, H_{\beta_{0}}, x, w_{0}\right) \\
& \quad \geq \int_{I_{\delta} \backslash U_{\delta}}\left|f_{1}(s, x) w_{0}(s)\right| d s+\beta_{0} \mathbb{I}\left(U_{\delta}, H_{\beta_{0}}, x, 1\right) \\
& \geq \int_{I_{\delta} \backslash U_{\delta}}\left|f_{1}(s, x) w_{0}(s)\right| d s+\beta_{0} \mathbb{I}\left(U_{\delta}, U_{\gamma_{1}}, x, 1\right) .
\end{aligned}
$$

By (3.2) from the last inequality (3.1) immediately follows.

Let $u_{n} \in \widetilde{C}^{\prime}(I ; R),\left\|u_{n}\right\|_{C} \neq 0(n \in N), w_{1}$ be an arbitrary nonzero solution of problem (1.3)-(1.4), and $r>0$. Then we define:

$$
\Omega_{w_{1}}^{+} \stackrel{\text { def }}{=}\left\{t \in I: w_{1}(t)>0\right\}, \quad \Omega_{w_{1}}^{-} \stackrel{\text { def }}{=}\left\{t \in I: w_{1}(t)<0\right\},
$$




$$
\begin{aligned}
& A_{n, 2} \stackrel{\text { def }}{=}\left\{t \in I:\left|u_{n}(t)\right| \leq r\right\}, \quad A_{n, 1} \stackrel{\text { def }}{=}\left\{t \in I:\left|u_{n}(t)\right|>r\right\}, \\
& B_{n, \ell} \stackrel{\text { def }}{=}\left\{t \in A_{n, 1}: \operatorname{sgn} u_{n}(t)=(-1)^{\ell-1} \operatorname{sgn} w_{1}(t)\right\} \quad(\ell=1,2) .
\end{aligned}
$$

From these definitions it is clear that, for all $n \in N$, we have

$$
\begin{array}{ll}
A_{n, 2} \cap A_{n, 1}=\emptyset, & A_{n, 2} \cup A_{n, 1}=I, \\
B_{n, 1} \cap B_{n, 2}=\emptyset, & B_{n, 1} \cup B_{n, 2}=A_{n, 1} \backslash N_{p} \subset I .
\end{array}
$$

Lemma 2. Let $r>0$, the functions $u_{n} \in \widetilde{C}^{\prime}(I ; R), n \in N$ admit to the conditions

$$
\begin{aligned}
& u_{n}(a)=0, \quad u_{n}(b)=0, \\
& \left\|u_{n}\right\|_{C}>2 r n \quad \text { for } \quad n \in N,
\end{aligned}
$$

and $w_{1}$ be such nonzero solution of problem (1.3)-(1.4), that the condition

$$
\left\|v_{n}^{(j)}-w_{1}^{(j)}\right\|_{C} \leq 1 / 2 n \quad \text { for } \quad n \in N(j=0,1)
$$

holds where $v_{n}(t)=u_{n}(t)\left\|u_{n}\right\|_{C}^{-1}$. Then for an arbitrary $\left.\delta \in\right] 0, \frac{1}{2} \min \left\{t_{1}-\right.$ $\left.a, b-t_{k}\right\}[$, and $\gamma \in] 0, \delta\left[\right.$ there exists $n_{0} \in N$ such that

$$
\begin{aligned}
& I_{\delta} \backslash U_{\gamma} \subset \cap_{n=n_{0}}^{+\infty} B_{n, 1}, \\
& B_{n, 2} \subset U_{\gamma} \text { for } n>n_{0}, \\
& \pm u_{n}(t) \geq r \quad \text { for } \quad t \in \Omega_{w_{1}}^{ \pm} \cap\left(I_{\delta} \backslash U_{\delta}\right), \quad n>n_{0} .
\end{aligned}
$$

Moreover

$$
\begin{aligned}
& \lim _{n \rightarrow+\infty} \operatorname{mes} A_{n, 2}=0, \quad \lim _{n \rightarrow+\infty} \operatorname{mes} A_{n, 1}=\operatorname{mes} I, \\
& \lim _{n \rightarrow+\infty} \operatorname{mes} B_{n, 2}=0, \quad \lim _{n \rightarrow+\infty} \operatorname{mes} B_{n, 1}=\operatorname{mes} I, \\
& \lim _{\delta \rightarrow 0} \operatorname{mes}\left(\Omega_{w_{1}}^{ \pm} \backslash\left[\Omega_{w_{1}}^{ \pm} \cap\left(I_{\delta} \backslash U_{\delta}\right)\right]\right)=0 .
\end{aligned}
$$

Proof. First note that from the unique solvability of Cauchy problem for equation (1.3) it is clear that

$$
w_{1}^{\prime}(a) \neq 0, \quad w_{1}^{\prime}(b) \neq 0 .
$$

Therefore conditions (3.6) and (3.8) imply the existence of such

$$
\delta \in] 0,1 / 2 \min \left\{t_{1}-a, b-t_{k}\right\}\left[\text {, and } n^{*} \in N\right. \text {, }
$$

that $\operatorname{sgn} w_{1}(t)=\operatorname{sgn} u_{n}(t)$ if $t \in[a, a+\delta] \cup[b-\delta, b]=I \backslash I_{\delta}$, and $n>n^{*}$. Consequently

$$
\left(I \backslash I_{\delta}\right) \cap B_{n, 2}=\emptyset \text { for } n>n^{*} .
$$

Now fix $\gamma \in] 0, \delta\left[\right.$, and note that $I_{\delta} \backslash U_{\gamma}$ is the closed set and $\min \left\{\left|w_{1}(t)\right|: t \in\right.$ $\left.I_{\delta} \backslash U_{\gamma}\right\}>0$. Therefore we can choose $n_{0}>n^{*}$ such that the inequality

$$
\left|w_{1}(t)\right| \geq 1 / n_{0} \quad \text { for } \quad t \in I_{\delta} \backslash U_{\gamma}
$$


holds, from which by (3.8) we obtain

$$
\begin{aligned}
& \left|v_{n}(t)\right| \geq 1 / 2 n_{0} \quad \text { for } \quad n \geq n_{0}, t \in I_{\delta} \backslash U_{\gamma}, \\
& \operatorname{sgn} w_{1}(t)=\operatorname{sgn} u_{n}(t) \quad \text { for } \quad n \geq n_{0}, t \in I_{\delta} \backslash U_{\gamma} .
\end{aligned}
$$

Also from (3.16) by (3.7) we get

$$
\left|u_{n}(t)\right|>n r / n_{0} \geq r \quad \text { for } \quad n \geq n_{0}, t \in I_{\delta} \backslash U_{\gamma} .
$$

From the last two relations follows that if $t \in I_{\delta} \backslash U_{\gamma}$ then $t \in B_{n, 1}$ for $n>n_{0}$, i.e. (3.9) holds. Now assume that there exists such increasing sequence $\left\{n_{j}\right\}_{j=1}^{+\infty}$ that $t_{n_{j}}^{\prime} \in B_{n_{j}, 2}$ and $t_{n_{j}}^{\prime} \notin U_{\gamma}$. Then taking into account (3.9), and (3.15) we get $t_{n_{j}}^{\prime} \in I \backslash\left(I \backslash I_{\delta}\right) \backslash U_{\gamma}=I_{\delta} \backslash U_{\gamma} \subset B_{n_{j}, 1}$ if $n>n_{0}$. But this is the contradiction with (3.5), i.e. (3.10) is valid.

For arbitrary $t^{*} \in I$ where $w_{1}\left(t^{*}\right) \neq 0$, in view of condition (3.8) the inequality $\left|w_{1}\left(t^{*}\right)-v_{n}\left(t^{*}\right)\right| \leq \frac{1}{2}\left|w_{1}\left(t^{*}\right)\right|$ holds if $n \geq 1 /\left|w_{1}\left(t^{*}\right)\right|$, i.e., $t^{*} \in B_{n, 1}$ if $n \geq 1 /\left|w_{1}\left(t^{*}\right)\right|$. Then the second equality of (3.13) holds, from which by (3.5) it follows the validity of first relation of (3.13) and validity of the second equality of (3.12). Then by (3.5) it is evident that the first equality of (3.12) holds also.

Inequality (3.11) immediately follows from (3.17) and (3.18). From the trivial relations $\lim _{\delta \rightarrow 0}$ mes $I_{\delta}=b-a, \lim _{\delta \rightarrow 0}$ mes $U_{\delta}=0$ it follows (3.14).

Now introduce the notation

$$
\mathbb{M}_{n}\left(w_{1}\right) \stackrel{\text { def }}{=} \int_{a}^{b}\left(h_{1}(s)+f_{1}\left(s, u_{n}(s)\right)\right) w_{1}(s) d s,
$$

then the following lemma is true

Lemma 3. Let the functions $u_{n}, w_{1}$ be such that all the assumptions of Lemma 2 are fulfilled, $r>0, f_{1} \in E\left(N_{p}\right)$, and there exist functions $f^{-}, f^{+} \in L\left(I, R_{+}\right)$ such that the conditions

$$
\begin{gathered}
f_{1}(t, x) \leq-f^{-}(t) \quad \text { for } \quad x \leq-r, \quad t \in I, \\
f^{+}(t) \leq f_{1}(t, x) \quad \text { for } \quad x \geq r, \quad t \in I,
\end{gathered}
$$

are satisfied. Let moreover there exists $\varepsilon>0$ such that for an arbitrary solution $w$ of problem (1.3)-(1.4) the condition

$$
\begin{gathered}
-\int_{a}^{b}\left(f^{+}(s)[w(s)]_{-}+f^{-}(s)[w(s)]_{+}\right) d s+\varepsilon \gamma_{r}\|w\|_{C} \leq-\int_{a}^{b} h_{1}(s) w(s) d s \\
\leq \int_{a}^{b}\left(f^{-}(s)[w(s)]_{-}+f^{+}(s)[w(s)]_{+}\right) d s-\varepsilon \gamma_{r}\|w\|_{C}
\end{gathered}
$$

holds, where $\gamma_{r}=\int_{a}^{b} f_{1}^{*}(t, r) d s$. Then there exists $n_{0} \in N$ such that

$$
\mathbb{M}_{n}\left(w_{1}\right) \geq 0 \quad \text { for } \quad n \geq n_{0} .
$$


Proof. First note that if $f_{1} \equiv 0$, then $\gamma_{r} \equiv f^{-} \equiv f^{+} \equiv 0$, and therefore in view of conditions (3.12) and (3.14) for arbitrary $\varepsilon>0$ there exist $\delta \in$ ]0, $\frac{1}{2} \min \left\{t_{1}-a, b-t_{k}\right\}\left[\right.$, and $n_{1} \in N$, such that

$$
\begin{aligned}
& \int_{A_{n, 2}} f^{*}(s, r) d s \leq \frac{1}{3} \varepsilon \gamma_{r} \text { for } n>n_{1} \\
& \frac{1}{\left\|w_{1}\right\|_{C}} \int_{\Omega_{w_{1}}^{ \pm}} f^{ \pm}(s)\left|w_{1}(s)\right| d s-\frac{1}{3} \varepsilon \gamma_{r} \leq \frac{1}{\left\|w_{1}\right\|_{C}} \int_{\Omega_{w_{1}}^{ \pm} \cap\left(I_{\delta} \backslash U_{\delta}\right)} f^{ \pm}(s)\left|w_{1}(s)\right| d s .
\end{aligned}
$$

Also, by the definition of the sets $A_{n, j}, B_{n, j}(j=1,2)$, and conditions (3.5) and (3.19), we obtain respectively the estimates

$$
\begin{aligned}
& \mathbb{M}_{n}\left(w_{1}\right) \geq-\int_{A_{n, 2}} f^{*}(s, r)\left|w_{1}(s)\right| d s+\int_{a}^{b} h_{1}(s) w_{1}(s) d s \\
& \quad+\int_{B_{n, 1}} f_{1}\left(s, u_{n}(s)\right) w_{1}(s) d s-\int_{B_{n, 2}}\left|f_{1}\left(s, u_{n}(s)\right) w_{1}(s)\right| d s, \\
& f_{1}\left(s, u_{n}(s)\right) w_{1}(s) \geq 0 \quad \text { for } \quad s \in B_{n, 1},
\end{aligned}
$$

for $n \in N$. On the other hand by Lemma 1 for chosen $\delta$ there exists $\gamma \in] 0, \delta[$ such, that inequality (3.1) holds, and now for chosen $\delta$, and $\gamma$, by Lemma 3.2 we can find $n_{0}>n_{1}$ such, that (3.11), and inclusions (3.9), (3.10), hold. Then from (3.23) in view of (3.1), by virtue of (3.9), (3.10), and (3.24), we get

$$
\begin{gathered}
\mathbb{M}_{n}\left(w_{1}\right) \geq-\int_{A_{n, 2}} f^{*}(s, r) d s\left\|w_{1}\right\|_{C}+\int_{a}^{b} h_{1}(s) w_{1}(s) d s \\
+\int_{I_{\delta} \backslash U_{\delta}} f_{1}\left(s, u_{n}(s)\right) w_{1}(s) d s \quad \text { for } \quad n \geq n_{0} .
\end{gathered}
$$

From the condition $N_{p} \neq \emptyset$ we obviously have $\Omega^{+} \neq \emptyset, \Omega^{-} \neq \emptyset, \Omega_{w_{1}}^{-} \cup \Omega_{w_{1}}^{+}=$ $\left(I \backslash N_{p}\right) \backslash\{a, b\}$, and then from (3.11), and (3.19), we get

$$
\begin{aligned}
& \quad \int_{I_{\delta} \backslash U_{\delta}} f_{1}\left(s, u_{n}(s)\right) w_{1}(s) d s=\int_{\Omega_{w_{1}}^{+} \cap\left(I_{\delta} \backslash U_{\delta}\right)} f_{1}\left(s, u_{n}(s)\right)\left|w_{1}(s)\right| d s-\int_{\Omega_{w_{1}}^{-} \cap\left(I_{\delta} \backslash U_{\delta}\right)} f_{1}\left(s, u_{n}(s)\right)\left|w_{1}(s)\right| d s \\
& \geq \int_{\Omega_{w_{1}}^{+} \cap\left(I_{\delta} \backslash U_{\delta}\right)} f^{+}(s)\left|w_{1}(s)\right| d s+\int_{\Omega_{w_{1}}^{-} \cap\left(I_{\delta} \backslash U_{\delta}\right)} f^{-}(s)\left|w_{1}(s)\right| d s,
\end{aligned}
$$

for $n>n_{0}$. Also it is clear that under the conditions of our Lemma equality (1.7) holds, and then

$$
\int_{\Omega_{w_{1}}^{ \pm}} f^{ \pm}(s)\left|w_{1}(s)\right| d s=\beta \int_{\Omega_{w_{1}}^{ \pm}} f^{ \pm}(s)[w(s)]_{ \pm} d s=\beta \int_{a}^{b} f^{ \pm}(s)[w(s)]_{ \pm} d s
$$

if $\beta>0$, and

$$
\int_{\Omega_{w_{1}}^{ \pm}} f^{ \pm}(s)\left|w_{1}(s)\right| d s=|\beta| \int_{\Omega_{w_{1}}^{ \pm}} f^{ \pm}(s)[w(s)]_{\mp} d s=|\beta| \int_{a}^{b} f^{ \pm}(s)[w(s)]_{\mp} d s
$$


if $\beta<0$. Then from (3.25) by virtue of (3.26), (3.22), and the last equalities we get

$$
\begin{aligned}
& \frac{\mathbb{M}_{n}\left(w_{1}\right)}{|\beta|} \geq-\varepsilon \gamma_{r}\|w\|_{C}+\int_{a}^{b}\left(f^{+}(s)[w(s)]_{+}+f^{-}(s)[w(s)]_{-}\right) d s \\
& \quad+\int_{a}^{b} h_{1}(s) w(s) d s \text { for } n \geq n_{0}, \beta>0, \\
& \frac{\mathbb{M}_{n}\left(w_{1}\right)}{|\beta|} \geq-\varepsilon \gamma_{r}\|w\|_{C}+\int_{a}^{b}\left(f^{+}(s)[w(s)]_{-}+f^{-}(s)[w(s)]_{+}\right) d s \\
& \quad-\int_{a}^{b} h_{1}(s) w(s) d s \text { for } n \geq n_{0}, \beta<0 .
\end{aligned}
$$

From the last inequalities by (3.20) we immediately obtain (3.21).

Lemma 4. Let the functions $u_{n}, w_{1}$ be such that all the assumptions of Lemma 2 are fulfilled, $r_{0}>0, f \in E\left(N_{p}\right)$, and

$$
f_{1}(t, x) \operatorname{sgn} x \geq 0 \quad \text { for } \quad|x| \geq r_{0}, \quad t \in I .
$$

Let moreover there exist such sets $I^{+}, I^{-} \subset I$, that

$$
\lim _{x \rightarrow \pm \infty}\left|f_{1}(t, x)\right|=+\infty \quad \text { uniformly on } I^{ \pm}
$$

and for an arbitrary solution $w$ of problem (1.3)-(1.4) inequalities (2.5) be satisfied. Then inequality (3.21) holds.

Proof. Let $h_{1} \in L(I, R)$ be arbitrarily chosen function, and $f^{ \pm}(t)=c$, for $t \in I^{ \pm}$, and 0 , for $t \in I \backslash I^{ \pm}$, where $c$ is a positive constant. In view of item a. of Remark 1, and conditions (2.5) we can find such $c>0$ that the inequality

$$
\begin{gathered}
-\int_{a}^{b}\left(f^{+}(s)[w(s)]_{-}+f^{-}(s)[w(s)]_{+}\right) d s<-\int_{a}^{b} h_{1}(s) w(s) d s \\
<\int_{a}^{b}\left(f^{-}(s)[w(s)]_{-}+f^{+}(s)[w(s)]_{+}\right) d s
\end{gathered}
$$

will hold, and for this $c$ in view of conditions (3.27) and (3.28) we can find such $r>0$, that inequalities (3.19) will be fulfilled. Also in view of item a. of Remark 1 it is clear that from (3.29), for given $r>0$, we can find such $\varepsilon>0$ that inequality (3.20) of Lemma 3 will be satisfied. Therefore for arbitrarily chosen $h_{1}$ all the conditions of Lemma 3 are fulfilled and then inequality (3.21) holds.

Lemma 5. Let $i \in\{0,1\}, p \in L(I ; R), p_{n}(t)=p(t)+(-1)^{i} / n$, and $w_{n} \in$ $\widetilde{C}^{\prime}(I ; R)(n \in N)$ be a solution of the problem

$$
w_{n}^{\prime \prime}(t)=p_{n}(t) w_{n}(t) \quad \text { for } \quad t \in I, \quad w_{n}(a)=0, \quad w_{n}(b)=0 .
$$

Then there exists $n_{1} \in N$ such that problem $\left(3.30_{n}\right)$ has only the zero solution for $n \geq n_{1}$. 
Proof. Let integers $A_{n} \geq 2$ and $N_{p}^{*} \geq 2$ be the number of zeros on $I$ of the nonzero solutions of problems $\left(3.30_{n}\right)$ and $(1.3),(1.4)$ respectively. Now assume on the contrary that there exists a sequence $\left\{w_{n}\right\}_{n \geq n_{1}}^{+\infty}$ of nonzero solutions of problem $\left(3.30_{n}\right)$. Then:

a. If $i=1$, from the fact that $p_{n}(t)<p_{n+1}(t)$ by Sturm's comparison theorem, we obtain $A_{n}-A_{n+k} \geq 1(n, k \in N)$. Now assume that $A_{n_{1}}=k_{1}$. Therefore, we obtain the contradiction $k_{1}=A_{n_{1}}>A_{n_{1}}-A_{n_{1}+k_{1}}=\left(A_{n_{1}}-\right.$ $\left.A_{n_{1}+1}\right)+\left(A_{n_{1}+1}-A_{n_{1}+2}\right)+\ldots+\left(A_{n_{1}+k_{1}-1}-A_{n_{1}+k_{1}}\right) \geq k_{1}$.

b. If $i=0$, from the fact that $p_{n-1}(t)>p_{n}(t)>p(t)$, by Sturm's comparison theorem, we obtain $A_{n}-A_{n-1} \geq 1$ and $N_{p}^{*} \geq A_{n}-1(n \in N)$. Therefore, if we denote $N_{p}^{*}=k_{1}$, we obtain the contradiction $k_{1}=N_{p}^{*} \geq A_{n+k_{1}}-1>$ $A_{n+k_{1}}-A_{n} \geq k_{1}$.

The contradictions obtained prove our lemma.

\section{Proof of the main results}

Proof of Theorem 1. Let $p_{n}(t)=p(t)+(-1)^{i} / n$ and for $n \in N$, consider the problems

$$
\begin{aligned}
u_{n}^{\prime \prime}(t) & =p_{n}(t) u_{n}(t)+f\left(t, u_{n}(t)\right)+h(t) \quad \text { for } \quad t \in I \\
u_{n}(a) & =0, \quad u_{n}(b)=0
\end{aligned}
$$

and $\left(3.30_{n}\right)$. In view of Lemma 5 , problem $\left(3.30_{n}\right)$ has only the zero solution for every $n \geq n_{1}$. Therefore, as it is well-known (see [9, Theorem 1.1, p. 345]), from the inequality (2.2) it follows that problem (4.1)-(4.2) has at least one solution, suppose $u_{n}$. Assume

$$
\lim _{n \rightarrow+\infty}\left\|u_{n}\right\|_{C}=+\infty
$$

and $v_{n}(t)=u_{n}(t)\left\|u_{n}\right\|_{C}^{-1}$. Then $v_{n}$ is the solution of the equation

$$
\left.v_{n}^{\prime \prime}(t)=p_{n}(t) v_{n}(t)+\frac{1}{\left\|u_{n}\right\|_{C}}\left(f\left(t, u_{n}(t)\right)\right)+h(t)\right),
$$

and the conditions

$$
\begin{aligned}
& v_{n}(a)=0, \quad v_{n}(b)=0, \\
& \left\|v_{n}\right\|_{C}=1,
\end{aligned}
$$

hold for $n \in N$. Hence, by the conditions (4.6) and (2.2), from (4.4) we get the existence of $r_{0}>0$ such that $\left\|v_{n}^{\prime}\right\|_{C} \leq r_{0}$. Consequently in view of (4.6), by Arzela-Ascoli lemma, without loss of generality we can assume that there exists $w_{1} \in \widetilde{C}^{\prime}(I, R)$ such that $\lim _{n \rightarrow+\infty} v_{n}^{(j)}(t)=w_{1}^{(j)}(t) \quad(j=0,1)$ uniformly on $I$. From the last equality and (4.3) there follows the existence of an increasing sequence $\left\{\alpha_{k}\right\}_{k=1}^{+\infty}$ of a natural numbers, such that $\left\|u_{\alpha_{k}}\right\|_{C}>2 r k$ and $\| v_{\alpha_{k}}^{(j)}-$ $w_{1}^{(j)} \|_{C} \leq 1 / 2 k$ for $k \in N$. Without loss of generality we can suppose that $u_{n} \equiv$ $u_{\alpha_{n}}$ and $v_{n} \equiv v_{\alpha_{n}}$. Then $u_{n}$ and $v_{n}$ are the solutions of problems (4.1)-(4.2) 
and (4.4)-(4.5) respectively, with $p_{n}(t)=p(t)+(-1)^{i} / \alpha_{n}$ for $t \in I, n \in N$, and inequalities (3.7) and (3.8) are fulfilled.

From (4.4), by virtue of (4.5), (3.7), (3.8), and (2.2), we obtain that $w_{1}$ is a solution of the problem (1.3)-(1.4). Multiplying equations (4.1) and (1.3) respectively by $w_{1}$ and $-u_{n}$, and integrating their sum from $a$ to $b$, in view of conditions (4.2) and (1.4), we obtain

$$
(-1)^{i+1} \int_{a}^{b}\left(h(s)+f\left(s, u_{n}(s)\right)\right) w_{1}(s) d s=\frac{\left\|u_{n}\right\|_{C}}{\alpha_{n}} \int_{a}^{b} w_{1}(s) v_{n}(s) d s
$$

for $n \geq n_{1}$. On the other hand in view of condition (3.8) we have

$$
\lim _{n \rightarrow+\infty} \int_{a}^{b} w_{1}(s) v_{n}(s) d s=\int_{a}^{b} w_{1}^{2}(s) d s>0 .
$$

Therefore from the last inequality and (4.7) it follows the existence of such $n_{2}>n_{1}$ that

$$
(-1)^{i+1} \int_{a}^{b}\left(h(s)+f\left(s, u_{n}(s)\right)\right) w_{1}(s) d s>0 \quad \text { for } \quad n \geq n_{2} .
$$

Now note that, in view the conditions $f \in E\left(N_{p}\right),\left(2.1_{i}\right),\left(2.2_{i}\right),(4.2)$, and (3.7), (3.8), all the assumptions of Lemma 3 with $f_{1}(t, x)=(-1)^{i} f(t, x), h_{1}(t)=$ $(-1)^{i} h(t)$ are satisfied. Therefore, the inequality (3.21) is true, which contradicts (4.8) when $n \geq \max \left\{n_{0}, n_{2}\right\}$. This contradiction proves that (4.3) does not hold and thus there exists $r_{1}>0$ such that $\left\|u_{n}\right\|_{C} \leq r_{1}$ for $n \in N$. Consequently, from (4.1) and (4.2) it is clear that there exists $r_{1}^{\prime}>0$ such that $\left\|u_{n}^{\prime}\right\|_{C} \leq r_{1}^{\prime}$ and $\left|u_{n}^{\prime \prime}(t)\right| \leq \sigma(t)$ for $t \in I, n \in N$, where $\sigma(t)=$ $(1+|p(t)|) r_{1}+|h(t)|+f^{*}\left(t, r_{1}\right)$. Hence, by Arzela-Ascoli lemma, without loss of generality we can assume that there exists a function $u_{0} \in \widetilde{C}^{\prime}(I ; R)$ such that $\lim _{n \rightarrow+\infty} u_{n}^{(j)}(t)=u_{0}^{(j)}(t)(j=0,1)$ uniformly on $I$. Therefore, it follows from (4.1) and (4.2) that $u_{0}$ is a solution of the problem (1.1)-(1.2).

Proof of Theorem 2. The proof is the same as the proof of Theorem 1. The only difference is that we use Lemma 4 instead of Lemma 3.

\section{Conclusions}

In the end of our paper we would like to consider following two tasks for future studies of resonance problem (1.1)-(1.2). The first task is to answer the question about the role of the condition $f \in E\left(N_{p}\right)$, for solvability of problem (1.1)-(1.2). Is it possible or not to omit this condition or replace it by the "easier" or more "natural" condition. The second task is to find the conditions of unique solvability of resonance problem (1.1)-(1.2) for the case when $N_{p} \neq \emptyset$, because if $N_{p}=\emptyset$, then it is not difficult to find the conditions of the unique solvability of our resonance problem. 


\section{References}

[1] S. Ahmad. A resonance problem in which the nonlinearity may grow linearly. Proc. Amer. Math. Soc., 92(3):381-384, 1984. https://doi.org/10.2307/2044839.

[2] N. Azbelevand, V. Maksimov and L.F. Rakhmatullina. Introduction to the Theory of Functional Differential Equations. Nauka, Moscow, 1991.

[3] C. De Coster and P. Habets. Upper and Lower Solutions in the theory of ODE boundary value problems. Sprringer Wien, NewYork, 1996. https://doi.org/10.1007/978-3-7091-2680-6_1.

[4] P. Drabek. On the resonance problem with nonlinearity which has arbitrary linear growth. J. Math. Anal. Appl., 127(2):435-442, 1987. https://doi.org/10.1016/0022-247X(87)90121-1.

[5] R. Gaines and J Mawhin. Coincidence Degree and Nonlinear Differential Equations. Springer-Verlag. Berlin. Heidelberg. New York, 1977. https://doi.org/10.1007/BFb0089537.

[6] C. Ha and C. Kuo. On the solvability of a two point boundary value problem at resonance. Topol. Methods Nonlinear Anal., 1(3):295-302, 1993. https://doi.org/10.12775/TMNA.1993.021.

[7] C. Ha and C. Kuo. On the solvability of a two point boundary value problem at resonance II. Topol. Methods Nonlinear Anal., 11(3):159-168, 1998. https://doi.org/10.12775/TMNA.1998.010.

[8] R. Iannacci and M. Nkashama. Nonlinear boundary value problems at resonance. Proc. Amer. Math. Soc., 11(4):455-473, 1987. https://doi.org/10.1016/0362546X(87)90064-2.

[9] R. Iannacci and M. Nkashama. Nonlinear two point boundary value problems at resonance without Landesman-Lazer condition. Proc. Amer. Math. Soc., 106(4):943-952, 1989. https://doi.org/10.2307/2047278.

[10] R. Kannan, J. Nieto and M. Ray. A class of nonlinear boundary value problems without Landesman-Lazer condition. J. Math. Anal. Appl., 105(1):1-11, 1985. https://doi.org/10.1016/0022-247X(85)90093-9.

[11] I. Kiguradze and B. Shekhter. Singular boundary value problems for second order ordinary differential equations. J. Sov. Math., 43(2):2340-2417, 1988. https://doi.org/10.1007/BF01100361.

[12] E. Landesman and A. Lazer. Nonlinear perturbations of linear elliptic boundary value problems at resonance. J. Math. Mech., 19(7):609-623, 1970. https://doi.org/10.1512/iumj.1970.19.19052.

[13] S. Mukhigulashvili. The Dirichlet BVP the second order nonlinear ordinary differential equation at resonance. Italian J. Of Pure and Appl. Math., 2011(28):177-204, 2011.

[14] S. Mukhigulashvili. The mixed BVP for second order nonlinear ordinary differential equation at resonance. Math. Nachr., 290(2):393-400, 2017. https://doi.org/10.1002/mana.201500247. 